\title{
Utilização de Pericárdio Bovino no Sling Pubovaginal para o Tratamento da Incontinência Urinária de Esforço
}

\author{
The Use of Bovine Pericardium in the Pubovaginal Sling for the \\ Treatment of Stress Urinary Incontinence
}

\begin{abstract}
Eduardo Batista Cândido, Sérgio Augusto Triginelli, Agnaldo Lopes da Silva Filho, Maurício Bechara Noviello, Admário Silva Santos Filho, Lucas Barbosa da Silva
\end{abstract}

\begin{abstract}
RESUMO
Objetivo: avaliar os resultados do uso do pericárdio bovino na cirurgia de sling pubovaginal para o tratamento da incontinência urinária genuína de esforço.

Métodos: avaliação prospectiva de cinco pacientes, com diagnóstico de incontinência urinária genuina de esforço submetidas à cirurgia de sling pubovaginal com utilização de faixa de pericárdio bovino, no período de outubro/2001 a dezembro/2001. A média de idade foi de 48,2 $\pm 11,5$ anos (33-69 anos).

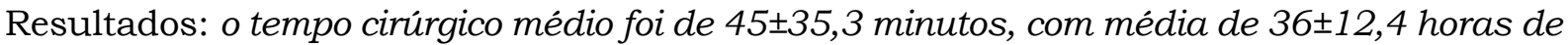
internação (24-48 horas). Não ocorreram complicações per-operatórias ou no periodo pósoperatório recente. Todas as pacientes apresentaram resultado inicial satisfatório, apresentando micções normais e sem perda. Ocorreram complicações pós-operatórias em todas as pacientes, sendo evidenciadas deiscência da ferida operatória vaginal com expulsão total da faixa em duas pacientes e exteriorização de parte da faixa em três pacientes. Todas as pacientes evoluíram com incontinência urinária de esforço, sendo submetidas à nova cirurgia de sling, com a utilização de fáscia do reto abdominal. Após a segunda cirurgia as pacientes evoluíram sem intercorrências e com melhora da perda involuntária de urina em quatro delas.

Conclusão: o sling pubovaginal com uso de pericárdio bovino associa-se a altas taxas de complicações, não devendo ser mais utilizado no tratamento da IUE.
\end{abstract}

PALAVRAS-CHAVE: Incontinência urinária. Urodinâmica. Cirurgia: complicações.

\section{Introdução}

Desde o início do século passado, os procedimentos do tipo sling têm sido descritos no tratamento da incontinência urinária de esforço (IUE), sendo utilizada grande variedade de materiais, como: músculo piramidal, fáscia lata, músculo grácil e bulbocavernoso $^{1}$. Em 1942, Aldridge ${ }^{2}$ utilizou uma faixa de fáscia do músculo reto abdominal, posicionada no nível do colo vesical, através de uma incisão vaginal ${ }^{2}$.

Departamento de Ginecologia e Obstetrícia da Faculdade de Medicina da Universidade Federal de Minas Gerais (UFMG); CENAGIN (Centro Avançado de Cirurgia Ginecológica), Hospital das Clínicas da UFMG

Correspondência:

Eduardo Batista Cândido

Avenida Pasteur, 89/1310 - Bairro Santa Efigênia

30150-290 - Belo Horizonte - MG

e-mail: agsilvaf@terra.com.br
Apesar das várias modificações técnicas, a cirurgia de sling consiste na utilização de uma faixa posicionada inferiormente à uretra ou colo vesical e fixada superiormente na parede abdominal. Apresenta taxas de cura objetiva de 61 a $100 \%$ e de cura subjetiva de 73 a $93 \%$ no tratamento da IUE feminina ${ }^{1}$.

As altas taxas de sucesso do sling suburetral no tratamento da IUE, associadas aos novos conceitos na fisiopatologia da IUE e ao desenvolvimento de técnicas menos invasivas com material sintético, têm ocasionado um ressurgimento do interesse pelo sling no tratamento da IUE feminina.

A utilização de tecido não autólogo no sling pubovaginal tem como vantagens a diminuição do tempo operatório, a possibilidade de utilização de anestesia local, além de menor tempo de internação e convalescência das pacientes ${ }^{1,3}$. Dessa forma, existe grande interesse por materiais 
biocompativeis que possam substituir as faixas autólogas na realização do sling, como a derme suína, a "lyodura" (homólogo liofilizado de duramáter), as faixas com polietileno, o Dácron ${ }^{\circledR}$, o polipropileno, o Gore-Tex ${ }^{\circledR}$, além do pericárdio bovino e o pericárdio de canguru ${ }^{3-6}$. No entanto, esses materiais heterólogos e sintéticos têm mostrado índices mais elevados de insucesso e complicações como rejeição e erosão uretral subseqüente $^{7,8}$. Existe ainda preocupação importante em relação à segurança do uso de materiais sintéticos em longo prazo ${ }^{9}$.

O pericárdio bovino é material biológico heterólogo de fácil manipulação e baixo custo, utilizado principalmente em cirurgias cardiovasculares, no tratamento de valvulopatias, aneurismas e dissecções arteriais, e nas neurocirurgias, nos enxertos na dura-máter ${ }^{10,11}$. Essas características motivaram a realização deste estudo para avaliar os resultados da utilização do pericárdio bovino no sling pubovaginal para o tratamento da incontinência urinária de esforço feminina.

\section{Pacientes e Métodos}

Foram estudadas de modo prospectivo, no período de outubro de 2001 a dezembro de 2001 , cinco pacientes portadoras de IUE submetidas à correção cirúrgica pela técnica de sling pubovaginal com utilização de faixa de pericárdio bovino. As pacientes foram avaliadas no pré-operatório com anamnese, exame físico e ginecológico e estudo urodinâmico completo. Foram excluídas do estudo pacientes que apresentavam instabilidade do detrusor. As pacientes assinaram o consentimento livre e esclarecido e o estudo foi aprovado pela Comissão de Ética em Pesquisa do Hospital das Clinicas da UFMG.

Utilizou-se o pericárdio bovino fornecido pela Biobrás Ltda. ${ }^{11}$, com $10 \times 6 \mathrm{~cm}$ de tamanho, tratado previamente com glutaraldeído em tampão de fosfato, purificado em carvão ativado e acondicionado em formaldeído. O pericárdio bovino era retirado da embalagem e lavado com solução salina antes do seu emprego.

A média de idade das pacientes foi de $48,2 \pm 11,5$ anos (33-69 anos), com paridade média de 3,8 $\pm 2,1$ partos (2-5 partos), sendo que uma paciente apresentava cesariana prévia. As pacientes não haviam sido submetidas previamente à cirurgia para correção da incontinência urinária. Todas as pacientes apresentavam perdas urinárias aos esforços, e os estudos urodinâmicos mostravam hipermotilidade uretral, a média da pressão de perda aos esforços foi de $96,7 \pm 23,2$ $\mathrm{cmH}_{2} \mathrm{O}\left(68-154 \mathrm{cmH}_{2} \mathrm{O}\right)$.
No pré-operatório imediato utilizou-se antibioticoprofilaxia com cefalotina, $2 \mathrm{~g}$, por via intravenosa. A paciente era posicionada em litotomia sob anestesia peridural, sendo realizado cateterismo vesical com sonda de Foley número 14. O sling era confeccionado com uma faixa de pericárdio bovino com 10 por $2 \mathrm{~cm}$, cujas bordas laterais eram suturadas com polipropileno 0 , mantendo longas as extremidades dos fios. Realizavase incisão longitudinal com $2 \mathrm{~cm}$ de extensão na mucosa vaginal anterior, distando $2 \mathrm{~cm}$ do meato uretral, seguida de dissecção lateral e identificação do espaço retro-púbico. A faixa era posicionada com auxílio de agulha de Raz no nível da uretra média, que era mantida sem tensão pela aproximação dos fios no plano mediano. Realizava-se síntese da mucosa vaginal e da pele.

O cateterismo vesical era retirado no primeiro dia de pós-operatório e a paciente recebia alta hospitalar após micção espontânea com volume residual inferior a $50 \mathrm{~mL}$. O seguimento era realizado em nível ambulatorial, com avaliação no sétimo dia de pós-operatório. A partir de então, a paciente era avaliada mensalmente nos primeiros seis meses e, a seguir, semestralmente.

\section{Resultados}

O tempo cirúrgico médio foi de $45 \pm 35,3$ minutos, com média de $36 \pm 12,4$ horas de internação (24-48 horas). Foi realizada perineoplastia posterior em duas pacientes. Não ocorreram complicações per-operatórias ou no período pós-operatório recente. Todas as pacientes apresentaram resultado inicial satisfatório, apresentando micções normais e sem perdas urinárias. Não houve retenção urinária nos casos estudados.

Ocorreram complicações pós-operatórias em todas as pacientes. As primeiras queixas de secreção vaginal purulenta ocorreram a partir do $28^{\circ}$ dia de pós-operatório (28-63 dias), sendo então evidenciada erosão e deiscência da ferida operatória vaginal. Ocorreu expulsão total da faixa em duas pacientes e exteriorização de parte da faixa em três pacientes. As pacientes evoluíram com infecção vaginal, com drenagem de copiosa secreção vaginal purulenta, sendo observada infecção do trato urinário em três casos (Tabela 1).

Realizou-se lavagem periódica da ferida vaginal com solução salina, sendo que aquelas pacientes com exteriorização parcial da faixa foram submetidas à retirada do pericárdio bovino e desbridamento da ferida vaginal no bloco cirúrgico. Nenhuma paciente apresentou erosão uretral 
ou vesical. Todas as pacientes evoluíram com incontinência urinária de esforço, sendo submetidas a nova cirurgia de sling, com a utilização de fáscia do reto abdominal. Esse novo procedimento foi difi- cultado pela presença de aderências no espaço retropúbico. Após a segunda internação as pacientes evoluiram sem intercorrências e com melhora da perda involuntária de urina em quatro casos.

Tabela 1 - Complicações pós-operatórias das pacientes submetidas à cirurgia de sling com pericárdio bovino.

Paciente

Erosão e deiscência
da ferida vaginal

\begin{tabular}{lll}
\hline 1 & Sim & Sim \\
2 & Sim & Não \\
3 & Sim & Não \\
4 & Sim & Sim \\
5 & Sim & Não \\
\hline
\end{tabular}

Complicações pós-operatórias

ITU: infecção do trato urinário. IUE: incontinência urinária de esforço.

\section{Discussão}

O sling pubovaginal autólogo consiste na retirada de um segmento fascial, do músculo reto abdominal ou fáscia lata, o que implica maior dificuldade técnica e aumento da morbidade, como dor pós-operatória, retenção urinária e maior tempo de internação ${ }^{12}$.

Os mais diversos materiais naturais e sintéticos têm sido utilizados na confecção da faixa do sling, dentre os quais citam-se: derme porcina, fáscias cadavéricas, faixas de propileno, Vycrilâ, silicone e Marlexâa, ${ }^{5,13}$. Um estudo utilizando corium suino mostrou permanência do material e ausência de complicações em $88 \%$ das 53 pacientes estudadas ${ }^{14}$.

Outro material heterólogo utilizado para a faixa do sling é a submucosa do intestino delgado suíno (SIS), que após processamento torna-se estrutura acelular formada por uma matriz de colágeno (tipos I, II e V) e fatores de crescimento (TGF-b e FGF-2). Um estudo com a utilização da SIS em 30 pacientes com IUE não mostrou qualquer sinal de infecção, inflamação ou rejeição no local da incisão vaginal ${ }^{15}$.

$\mathrm{Na}$ literatura existem poucos trabalhos avaliando a utilização de pericárdio bovino na cirurgia de sling e os resultados são controversos. Estudo envolvendo 22 pacientes com a utilização de pericárdio bovino para tratamento de IUE, com seguimento médio de 20 meses, não mostrou rejeição, erosão ou infecção ${ }^{16}$. No entanto, outro estudo, no qual foram avaliadas 10 pacientes por período médio de nove meses, mostrou exteriori- zação da faixa em $50 \%$ das pacientes, erosão em $10 \%$ das pacientes, incontinência persistente em $20 \%$ das pacientes, infecção urinária em 10\% das pacientes e infecção de parede abdominal em 10\% das pacientes ${ }^{17}$.

Neste trabalho, a utilização de pericárdio bovino teve como complicação a rejeição em todas as pacientes, evidenciada por deiscência da ferida vaginal com exteriorização parcial da faixa em três de cinco pacientes e expulsão total em duas pacientes. Essas altas taxas de insucesso parecem estar relacionadas ao tecido heterólogo utilizado, já que a nossa experiência com o sling pubovaginal com fáscia do reto abdominal não resulta em índices de complicações tão expressivos.

São incertas as razões específicas que justificariam a rejeição, podendo estar relacionadas com a preservação do pericárdio bovino e sua esterilização. A rejeição poderia ser causada pela antigenicidade desse tecido, ocasionando reação do tipo corpo estranho. O local de sua utilização parece ter importância, já que o pericárdio bovino é utilizado em outros sítios com boa tolerância. No entanto, quando em contato com a mucosa vaginal haveria contaminação bacteriana e conseqüente rejeição. Estudo utilizando fáscia lata cadavérica no sling mostrou altos índices de rejeição, apesar de esse material ser utilizado em outras cirurgias ${ }^{18}$.

Concluímos que o pericárdio bovino não é tecido recomendável para a confecção da faixa no sling pubovaginal. A alta taxa de rejeição torna proibitivo o seu uso no tratamento cirúrgico da incontinência urinária de esforço. 


\section{ABSTRACT}

Purpose: to evaluate the results of the use of bovine pericardium in the pubovaginal sling procedure for treatment of stress urinary incontinence.

Methods: a prospective analysis of five patients who underwent pubovaginal sling with the use of bovine pericardium strip for stress urinary incontinence in the "Hospital das Clínicas of UFMG" from October/2001 to December/2001. The mean age was $48.2 \pm 11.5$ years (33 to 69 years).

Results: the mean surgical time was $45 \pm 35.3$ min and the mean hospital stay was $36 \pm 12.4 \mathrm{~h}$ ( 24 to $48 \mathrm{~h}$ ). Complications in the periperative or immediate postoperative period did not occur. All patients initially presented satisfactory results with normal voiding and without stress incontinence. Postoperative complications occurred in the 5 patients (100\%), with dehiscence of the vaginal wound and total expulsion of the strip in 2 patients (40\%) and partial expulsion in 3 patients (60\%). All patients presented stress urinary incontinence and were submitted to a new sling procedure using the rectus fascia. The patients then progressed without complications and with improvement of urinary continence in 4 patients (80\%).

Conclusion: pubovaginal sling with the use of bovine pericardium was associated with high rates of complications. Therefore, its use is not recommended in the treatment of stress urinary incontinence.

KEYWORDS: Urinary incontinence. Urodynamics. Pubovaginal sling.

\section{Referências}

1. Bidmead J, Cardozo L. Sling techniques in the treatment of genuine stress incontinence. BJOG 2000; 107:147-56.

2. Aldridge AH. Transplantation of fascia for relief of urinary stress incontinence. Am J Obstet Gynecol 1942; 44:398-411.

3. Ghonheim G, Shaaban A. Suburethral slings for the treatment of stress urinary incontinence. Int Urogynecol J Pelvic Floor Dysfunct 1994; 5:228-39.

4. Appell RA. Primary slings for everyone with genuine stress incontinence? The argument for.... Int Urogynecol J Pelvic Floor Dysfunct 1998; 9:249-51.
5. Bent AE, Ostergard DR, Zwick-Zaffuto M. Tissue reaction to expanded polytetrafluoroethylene suburethral sling for urinary incontinence: clinical and histologic study. Am J Obstet Gynecol 1993; 169:1198-204.

6. Stanton SL, Brindley GS, Holmes DM. Silastic sling for urethral sphincter incontinence in women. Br J Obstet Gynaecol 1985; 92:747-50.

7. Bezerra CA, Bruschini H, Cody DJ. Suburethral sling operations for urinary incontinence in women. Cochrane Database Syst Rev 2003; (2):CD00185.

8. Norris JP, Breslin DS, Staskin DR. Use of synthetic material in sling surgery: a minimally invasive approach. J Endourol 1996; 10:227-30.

9. Bemelmans B. The use of biomaterials in incontinence surgery. Eur Urol 2002; 1:12-6.

10.Brick AV, Miana AA, Colen EA, et al. Seguimento de 9 anos da bioprótese valvar cardiaca de pericárdio bovino IMC- Biomédica; estudo multicêntrico. HU Rev 1987; 14:27-49.

11.Barbenal JC, Zioupos P, Fisher J. The mechanical properties of bovine pericardium. Heart Valve Eng 1986; 99:201-4.

12.Kuo HC. Comparison of video urodynamic results after the pubovaginal sling procedure using rectus fascia and polypropylene mesh for stress urinary incontinence. J Urol 2001; 165:163-8.

13. Melnick I, Lee RE. Delayed transection of urethra by mersilene tape. Urology 1976; 8:580-1.

14.Iosif CS. Porcine corium sling in the treatment of urinary stress incontinence. Arch Gynecol 1987; 240:131-6.

15.Palma PCR, Dambros M, Riccetto CLZ, Herrmann V, Rodrigues Júnior N. Pubovaginal sling using the porcine small intestine submucosa for stress urinary incontinence. Braz J Urol 2001; 27:483-8.

16.Pelosi MA $2^{\text {nd }}$, Pelosi MA $3^{\text {rd }}$, Pelekanos M. The YAMA UroPatch sling for treatment of female stress urinary incontinence: a pilot study. J Laparoendosc Adv Surg Tech A 2002; 12:27-33.

17. Martucci RC, Ambrogini A, Calado AA, Zerati M, Muller MA. Pubovaginal sling with bovine pericardium for treatment of stress urinary incontinence. Braz J Urol 2000; 26:208-14.

18.Huang YH, Lin AT, Chen KK, Pan CC, Chang LS. High failure rate using allograft fascia lata in pubovaginal sling surgery for female stress urinary incontinence. Urology 2001; 58:943-6. 